\title{
Comparison of three NIRS devices for the measurement of microvascular reactivity
}

Kevin Steenhaut MD, Stefan De Hert MD, PhD, Anneliese Moerman MD, PhD

Ghent University Hospital, Dept of Anaesthesiology, Ghent, Belgium

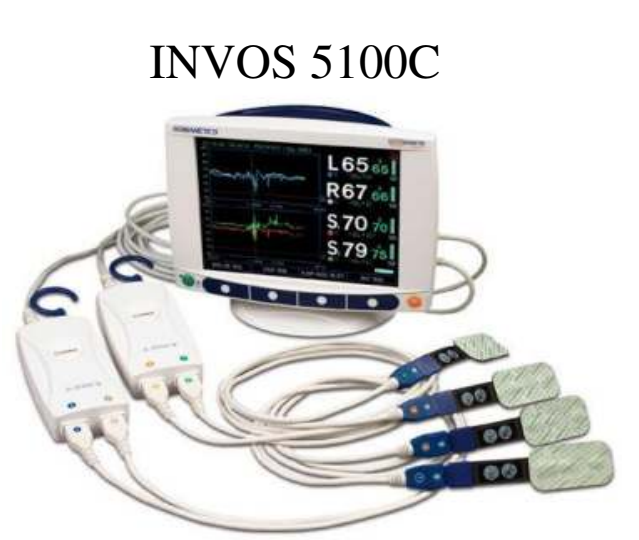

\section{Background}

- Near-infrared spectroscopy (NIRS) is increasingly recognized as a method for non-invasive assessment of microvascular reactivity.

- Comparability between different NIRS devices is unclear as there are several technical differences between them.

\section{$\underline{\text { Aim }}$}

To analyse and compare tissue oxygenation measurements by three different NIRS devices during a vascular occlusion test.

\section{Materials and methods}

- Forty consenting adults scheduled for elective coronary artery bypass grafting surgery.

- Three NIRS sensors, one of each device, were applied over the left brachioradial muscle.

- The different parameters of microvascular reactivity used for analysis, are shown in figure 1.

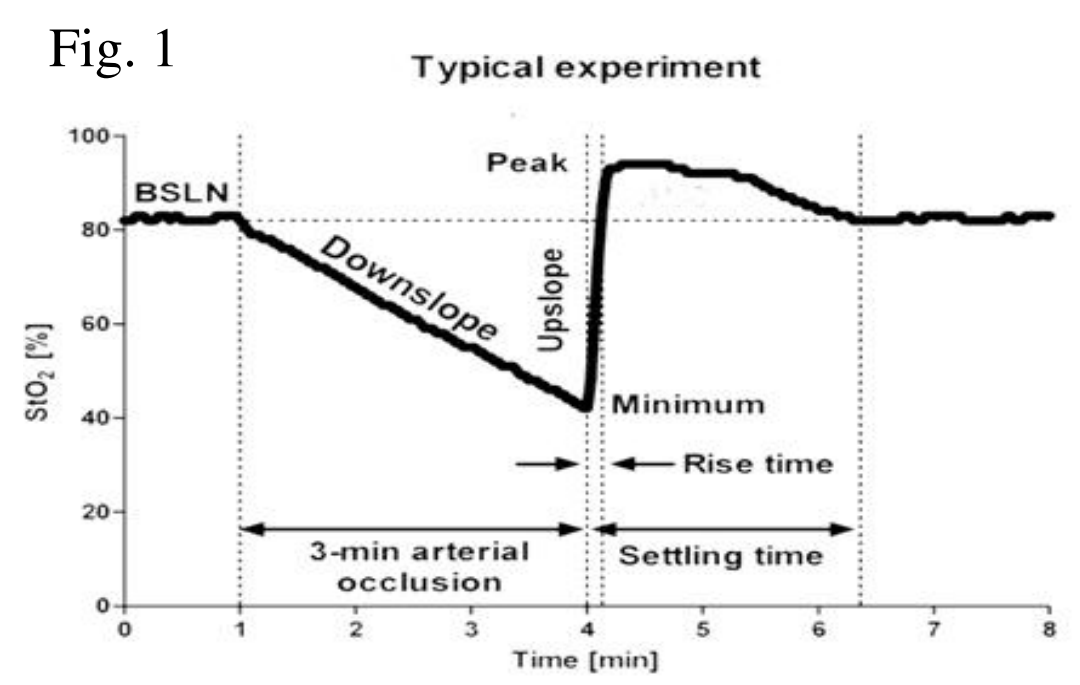

- Comparisons between devices were performed with the Kruskal-Wallis test. Pairwise differences among devices were examined with the MannWhitney U test.
FORESIGHT Elite

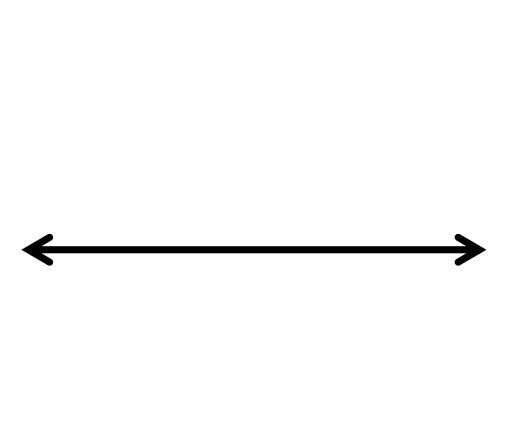

NIRO 200-NX

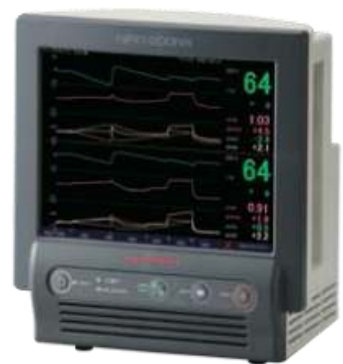

\section{$\underline{\text { Results }}$}

There were no significant differences at baseline. INVOS had significantly higher downsloping rates, lower minimum values and higher upsloping rates, while NIRO had lower maximum peak values and Foresight had longer rise and settling times compared to the two other devices.
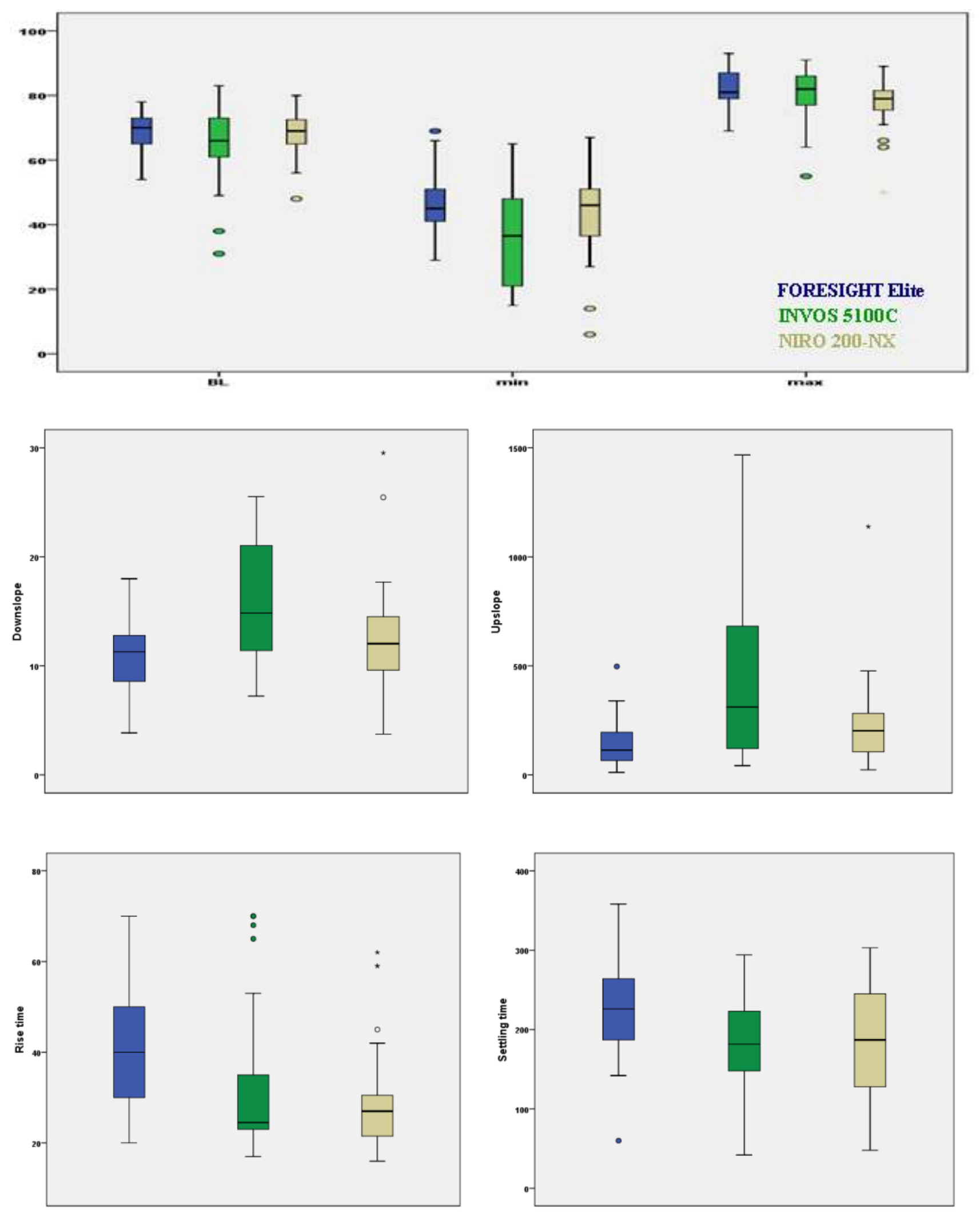

The boxes represent the interquartile ranges, the wiskers the range and the horizontal bar the median. Outliers are marked by dots.

\section{Conclusion}

Different information is retrieved depending on the NIRS device used. This phenomenon should be kept in mind when using NIRS for measurement of peripheral tissue oxygenation and when using NIRS values as threshold values in goal-directed treatment protocols. 